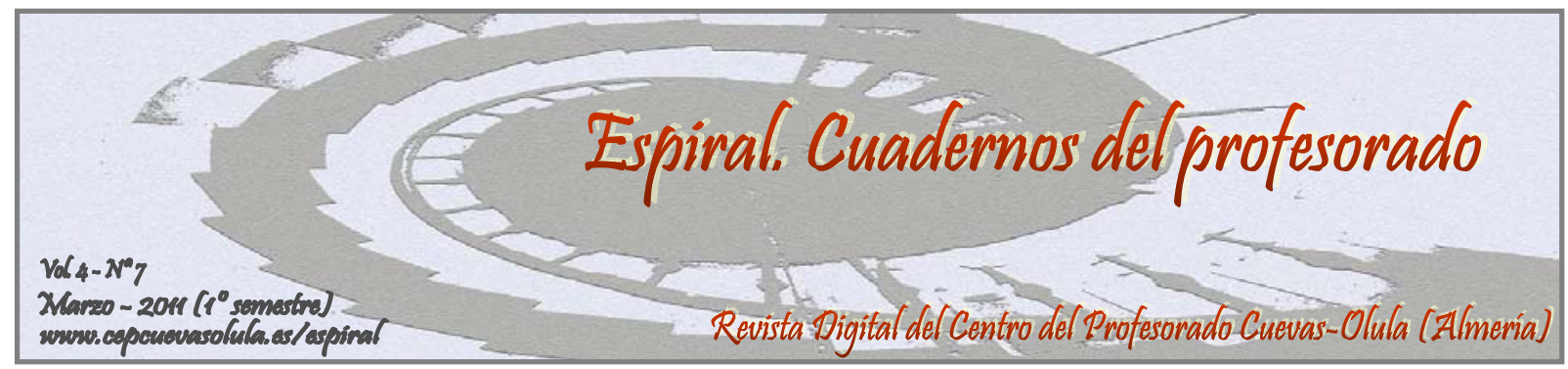

\title{
MOTIVOS DE PRÁCTICA EN EL ÁMBITO DE LA ACTIVIDAD FÍSICA NO COMPETITIVA
}

\section{MOTIVES OF PRACTICE IN THE FIELD OF NON-COMPETITIVE PHYSICAL ACTIVITY}

\author{
Antonio Granero-Gallegos ${ }^{(1)}$, Manuel Gómez-López ${ }^{(1)}$, \\ J. Arturo Abraldes Valeiras ${ }^{(1)}$ y Nuria Rodríguez-Suárez ${ }^{(2)}$,
}

\author{
${ }^{(1)}$ Facultad de Ciencias del Deporte, Universidad de Murcia, España \\ ${ }^{(2)}$ Facultad de Ciencias de la Salud, de la Actividad Física y del Deporte, Universidad Católica de \\ San Antonio de Murcia, España.
}

\begin{abstract}
RESUMEN: El objetivo del estudio fue conocer cuáles son los motivos de práctica en el ámbito de la actividad física no competitiva. Para ello se administró la escala de medición de los motivos de actividad física revisada (MPAM-R, Motives for Physical Activity Measure-Revised) de Ryan, Frederick, Lepes, Rubio y Sheldon (1997) y validada en España por Moreno, Cervelló y Martínez (2007) a una muestra compuesta por 271 sujetos adultos practicantes de actividades físicas no competitivas. Se realizó un análisis factorial de componentes principales de la escala y un análisis de correlación de las distintas subescalas. Los resultados manifiestan que las principales razones para realizar actividades físicas son el fomento de las relaciones sociales así como la obtención de placer y diversión, por delante de otras como la competencia, el mantenimiento y mejora de la salud y la apariencia.
\end{abstract}

Palabras clave: motivos, deporte, actividad física, salud, fitness.

ABSTRACT: The objective was to study what are the reasons of practice in the field of noncompetitive physical activity. To this end, the scale of measurement of physical activity based on revised (MPAM-R, Motives for Physical Activity Measure-Revised) Ryan, Frederick, Lepes, Rubio and Sheldon (1997) and validated in Spain by Moreno, Cervelló and Martínez (2007) was given to a sample of 271 adult subjects practicing uncompetitive physical activities. We conducted a principal components factor analysis of scale and a correlation analysis of the subscales. The results show that the main reasons for physical activity are the promotion of social relations and the achievement of pleasure and fun, ahead of other as competition, maintaining and improving health and appearance.

Key words: reasons, sport, physical activity, health, fitness

Granero-Gallegos, A., Gómez-López, M., Abraldes Valeiras, J.A. y Rodríguez-Suárez, N. (2011). Motivos de práctica en el ámbito de la actividad física no competitiva. Espiral. Cuadernos del Profesorado [en línea], 4(7), 15-22. Disponible en: http://www.cepcuevasolula.es/espiral. 


\section{1.- INTRODUCCIÓN.}

En el ámbito de la actividad física y el deporte la motivación se entiende como un proceso individual y dinámico bastante complejo en el que inciden diversas variables que interactúan entre sí y que puede utilizarse a la hora de explicar el comportamiento de las personas cuando participan en una actividad física, deportiva y recreativa (Roberts, 1992; SantosRosa, García, Jiménez, Moya \& Cervelló, 2007). Se trata del producto de un conjunto de variables variables sociales, ambientales e individuales que interactúan determinando la elección de una u otra actividad deportiva, la intensidad en la práctica de dicha actividad, la persistencia en la tarea y el rendimiento (Balaguer, 1994; Escartí \& Cervelló, 1994).

La motivación determina la dirección y la intensidad del esfuerzo, entendiendo por esfuerzo si el individuo busca o se siente atraído por ciertas situaciones y por intensidad a la cantidad de empeño que una persona emplea en una determinada situación (Escartí \& Brustad, 2002; Weinberg \& Gould, 2010). Tanto la intensidad como la dirección que adoptan los motivos de práctica son relevantes para la continuidad en una actividad física y/o deportiva (Cervelló, Escartí \& Guzmán, 2007; Ryska, Hohensee, Cooley \& Jones, 2002). De este modo, la motivación representa un elemento clave en la obtención del compromiso y la adherencia en el ámbito físico y/o deportivo, ya que su estudio nos permitirá conocer por qué algunas personas eligen una determinada actividad y los factores que determinan la permanencia o abandono de esta elección (Moreno, Cervelló \& Martínez, 2007). Bakker, Whiting \& Van der Brug (1990) definen motivo como la disposición de la persona y las razones que le llevan a hacer algo.

Actualmente en el ámbito del deporte y de la actividad física existe gran complejidad y diferenciación tanto en sus formas de organización como de formación y heterogeneidad en los motivos de práctica (García Ferrando, 2001), debido en gran parte a la apareciendo nuevas prácticas y tendencias físico-deportivas, cada vez más diversificadas (Palomares, 2005).

Según Cantón, Mayor y Pallarés (1995), para que una persona comience y mantenga la práctica de una actividad física o un deporte es necesario que posea algún atractivo. Masachs, Puente y Blasco (1994) afirman que la práctica regular conlleva la modificación sustancial de las motivaciones del sujeto, y determina la aparición de razones para el mantenimiento de la actividad física que no habían sido consideradas en el momento de iniciarlas.

En el estudio de los motivos se han utilizado como instrumentos de medida preferentemente los cuestionarios, que desde un doble enfoque, sociológico y psicológico, han proporcionado las bases para establecer las teorías de la motivación (Dosil, 2008). En nuestra investigación nos hemos decantado por la escala de Medida de Motivos de la Actividad Física Revisada (MPAM-R, Motives for Physical Activities Measure-Revised) de Ryan, Frederick, Lepes, Rubio y Sheldon (1997) que mide cinco áreas distintas como el fitness/salud, apariencia, social, competencia y disfrute.

Los resultados que se obtengan del estudio aportarán información valiosa a la gestión de los centros de actividad física no competitiva que les permitirá perfeccionar su oferta de actividades atendiendo a las preferencias y necesidades reales de los usuarios y a todas aquellas instituciones cuyo objetivo sea el fomento de la actividad física y de un estilo de vida saludable a través de campañas de promoción (Moreno et al., 2007).

Así pues, el propósito del presente estudio fue analizar los motivos de participación en el ámbito de la actividad física no competitiva de una muestra de personas procedentes de diferentes localidades de la Xunta de Galicia mediante la escala MPAM-R.

\section{2.- MÉTODO.}

\section{1.- Muestra.}

En este estudio han participado un total de 271 sujetos (edad: $M=18.90 ; D T=4.56$ ) que participaron en actividades físicas no competitivas. La muestra fue de 132 hombres $(M$ $=19.24 ; D T=5.06)$ y 139 mujeres $(M=18.57$; $D T=4.06$ ), procedentes de diferentes localidades de la Xunta de Galicia.

\section{2.- Instrumento.}

La escala Motives for Physical Activities Measure-Revised (MPAM-R), fue mostrada por Ryan et al. (1997), siendo una revisión y modificación de la escala Motives for Physical Activities Measure de Frederick-Recascino y Ryan (1993). Mide los motivos de práctica hacia la actividad física mediante 30 ítems, agrupados en cinco factores distintos: disfrute, apariencia, social, fitness/salud y competencia. De las cinco 
dimensiones, tanto disfrute como competencia han sido utilizadas para reflejar una orientación motivacional intrínseca, mientras que las otras tres subescalas (social, fitness/salud y apariencia) lo han sido para determinar niveles de motivación extrínseca (Ryan et al., 1997).

En la presente investigación se utilizó la versión en castellano de la escala, validada al contexto español por Moreno et al. (2007) en población de practicantes de actividades físicas no competitivas (musculación, aeróbic, actividades acuáticas, etc.). Se mide a través de ítems policotómicos en una escala tipo Likert de siete puntos: (1) corresponde a "nada verdadero para mí" y (7) a "totalmente verdadero para mí". Los valores de alfa de Cronbach obtenidos por Ryan et al. (1997) fueron de $\alpha=.92, \alpha=.91, \alpha=$ $.83, \alpha=.78 \alpha=.88$, respectivamente.

Esta escala ha sido utilizada y validada por otros autores en distintos contextos (FrederickRecascino y Schuster-Smith, 2003), obteniendo en todos ellos una adecuada fiabilidad y validez. En España, Moreno et al. (2007) obtuvieron los siguientes coeficientes de consistencia interna: disfrute, $\alpha=.84$; apariencia, $\alpha=.87$; social, $\alpha=$ .81 ; fitness, $\alpha=.80$; competencia, $\alpha=.85$

\section{3.- Procedimiento.}

Todos los participantes fueron informados del objetivo del estudio, voluntariedad, absoluta confidencialidad tanto de las respuestas como del manejo de los datos y de cómo cumplimentar el cuestionario, el cual fue administrado por los investigadores.

A los participantes se les insistió en los siguientes aspectos: que no había respuestas correctas o incorrectas, que no dejaran ningún ítem $\sin$ contestar y que lo hicieran con la máxima sinceridad y honestidad. La duración aproximada de la pasación del instrumento fue de 10 minutos. Se respetaron en todo momento todos los procedimientos éticos en la recogida de los datos.

\section{4.- Análisis de datos.}

En primer lugar se calcularon los estadísticos descriptivos y los índices de asimetría y curtosis siendo, en general, próximos a cero y con valores por debajo de dos, tal y como recomiendan Bollen y Long (1994), lo que indica semejanza con la curva normal de forma univariada y permite utilizar técnicas factoriales.

Este aspecto se ve reforzado con los cálculos obtenidos en las puntuaciones del estadístico de
Kolmogorov-Smirnov, obteniendo puntuaciones adecuadas ( $Z$ entre .79 y $.93 ; p>.09)$, asegurando normalidad en la distribución de la muestra. Se ha llevado a cabo un análisis factorial exploratorio (análisis de componentes principales con rotación Varimax con Kaiser), tomándose el coeficiente KMO (Kaiser-MeyerOlkin) como índice para analizar la adecuación del análisis factorial aplicado al grupo de variables analizadas, así como la prueba de esfericidad de Bartlett para las correlaciones positivas entre variables. Se calculó la fiabilidad de cada subescala propuesta originalmente por los autores a través del índice de consistencia interna alfa de Cronbach $(\alpha)$. Seguidamente se realizó un análisis de correlación entre todas las variables. Para obtener los resultados sobre los efectos principales de la interacción entre las distintas variables se realizó una MANOVA. El programa estadístico utilizado para el análisis ha sido el SPSS 17.0.

\section{3.- RESULTADOS.}

\section{1.- Propiedades psicométricas de la escala MPAM-R.}

Análisis factorial de componentes principales $(P C A)$. Con la muestra descrita se llevó a cabo un análisis factorial de componentes principales con rotación oblicua Varimax con Kaiser. Este PCA se realizó con 29 de los 30 ítems de la escala, pues fue eliminado el ítem 28 ("Para buscar la aceptación de los demás"), dado que esta dimensión no alcanzaba una adecuada consistencia interna con el mismo. Los 29 ítems se quedaron agrupados en cinco factores (tabla 1), denominados: disfrute, competencia, apariencia, fitness y social, con autovalores superiores a 1.00 y una varianza total explicada del $67.66 \%$. El coeficiente de visualización para la correlación mínima de las variables se situó en 40 (Pedhazur, 1982; Stevens, 1992). El coeficiente KMO que se ha obtenido es de .90 , lo que indica el alto grado de adecuación muestral, ya que el grado de variabilidad total de la muestra se explica al 90\%. Así mismo, el nivel crítico (sig. unilateral: .00) de la prueba de esfericidad de Bartlett muestra el alto grado de correlaciones positivas entre variables

Análisis de consistencia interna. La consistencia interna del instrumento se obtuvo calculando el coeficiente alfa de Cronbach $(\alpha)$. Se incluyó, además, la opción calcular $\alpha$ si era eliminado un elemento. 
Tabla 1.- Análisis factorial de la escala MPAM-R. Matriz de componentes rotados. Método de extracción: Análisis de componentes principales. Método de rotación: Normalización Varimax con Kaiser, coeficiente de visualización 0.40.

\begin{tabular}{|c|c|c|c|c|c|}
\hline & 1 & 2 & 3 & 4 & 5 \\
\hline \multicolumn{6}{|l|}{ Disfrute } \\
\hline 2. Porque es divertido & .78 & & & & \\
\hline 7. Porque me gusta hacer esta actividad & .83 & & & & \\
\hline 11. Porque me hace feliz & .72 & & & & \\
\hline 18. Porque pienso que es interesante & .59 & & & & \\
\hline 22. Porque disfruto con esta actividad & .74 & & & & \\
\hline 26. Porque encuentro esta actividad estimulante & .64 & & & & \\
\hline 29. Porque me gusta la excitación de participar & .65 & & & & \\
\hline
\end{tabular}

\section{Apariencia}

5. Porque quiero mantener mi peso para tener buena imagen $\quad .64$

10. Porque quiero definir mis músculos para tener buena imagen $\quad .77$

17. Porque quiero mejorar mi apariencia $\quad .52$

20. Porque quiero resultar atractivo a los demás $\quad .72$

24. Porque quiero mejorar mi cuerpo $\quad .66$

27. Porque me sentiré poco atractivo físicamente si no lo practico 82

\section{Social}

6. Porque me gusta estar con mis amigos

15. Porque me gusta estar con otros a los que también les interesa esta actividad

21. Porque quiero conocer a nuevas personas

30. Porque me gusta pasar el tiempo con otros haciendo esta actividad.

\section{Fitness/Salud}

1. Quiero estar en buena forma física

13. Quiero tener más energía

16. Quiero mejorar mi aptitud cardiovascular

19. Quiero mantener mi fuerza física para vivir de forma

\section{Competencia}

3. Porque me gusta comprometerme en actividades que físicamente suponen un desafío

4. Porque quiero desarrollar nuevas habilidades

8. Porque quiero mejorar las habilidades que poseo

9. Porque me gustan los desafíos

12. Porque quiero mantener mi nivel de habilidad actual

14. Porque me gustan las actividades que suponen un reto

25. Porque quiero estar bien para desarrollar mi actividad

$\begin{array}{rccccc}\text { Autovalor } & 4.36 & 4.01 & 3.74 & 3.56 & 3.12 \\ \text { \% varianza } & 21.03 & 15.70 & 11.01 & 10.45 & 9.47 \\ \text { arianza total } & & & & & 67.66\end{array}$

El factor 1 (disfrute), compuesto por siete ítems, estaba referido a la práctica de actividad física como actividad placentera y de diversión. Incluyó los siguientes ítems: "Porque es divertido", "Porque me gusta hacer esta actividad", "Porque me hace feliz", "Porque pienso que es interesante", "Porque disfruto con esta actividad", "Porque encuentro esta 
actividad estimulante", "Porque me gusta la excitación de participar". El coeficiente de fiabilidad de este factor fue de $\alpha=.84$.

El factor 2 (apariencia) lo componían seis ítems relacionados con la práctica de actividad física como medio para mejorar la imagen corporal y la apariencia externa. Estaba formado por: "Porque quiero mantener mi peso para tener buena imagen", "Porque quiero definir mis músculos para tener buena imagen", "Porque quiero mejorar mi apariencia", "Porque quiero resultar atractivo a los demás", "Porque quiero mejorar mi cuerpo", "Porque me sentiré poco atractivo fisicamente si no lo practico". El coeficiente de fiabilidad de este factor fue de $\alpha=$ .89 .

El factor 3 (social), lo componían cuatro factores relacionados con la práctica de actividad física como medio de establecer, mejorar o mantener las relaciones sociales. En la escala original este subescala estaba compuesta por cinco ítems, pero fue eliminado el número 28 , "Para buscar la aceptación de los demás", para que la consistencia interna de esta dimensión fuera considerada adecuada. Así, este factor se compuso por: "Porque me gusta estar con mis amigos", "Porque me gusta estar con otros a los que también les interesa esta actividad", "Porque quiero conocer a nuevas personas", "Porque me gusta pasar el tiempo con otros haciendo esta actividad". El coeficiente de fiabilidad de este factor fue de $\alpha=.77$.

El factor 4 (fitness/salud), referido a la práctica de actividades físicas como medio para mantener o mejorar la salud, estaba compuesto por cinco ítems: "Quiero estar en buena forma fisica", "Quiero tener más energía", "Quiero mejorar mi aptitud cardiovascular", "Quiero mantener mi fuerza física para vivir de forma saludable", "Quiero mantener mi salud física y mi bienestar". El coeficiente de fiabilidad de este factor fue de $\alpha=.80$.
El factor 5 (competencia), se relacionó con la práctica de actividad física para la mejora de habilidades o para realizar aquellas prácticas que suponían un desafío para el sujeto. Estaba formado por siete ítems: "Porque me gusta comprometerme en actividades que fisicamente suponen un desafio", "Porque quiero desarrollar nuevas habilidades", "Porque quiero mejorar las habilidades que poseo", "Porque me gustan los desafios", "Porque quiero mantener mi nivel de habilidad actual", "Porque me gustan las actividades que suponen un reto fisicamente", "Porque quiero estar bien para desarrollar mi actividad". El coeficiente de fiabilidad de este factor fue de $\alpha=.88$.

\section{2.- Descriptivos y análisis de correlación.}

En la tabla 2 se muestran los valores descriptivos de las variables estudiadas. La puntuación media de las subescalas social y disfrute $(M=5.85$ y 5.83 respectivamente) son claramente superiores al resto de las subescalas. Le siguen en orden de valoración la competencia $(M=5.68)$, fitness $(M$ $=5.57)$ y la apariencia $(M=4.45)$

Para comprobar las relaciones existentes entre las diferentes subescalas se realizó un análisis de correlaciones, que se presenta en la tabla 2. A nivel general, se observa una importante y significativa correlación positiva entre el disfrute y el resto de las subescalas analizadas, siendo mayor tanto con las motivos relacionados con lo social y la competencia $(r=$ $.79 ; \mathrm{p}<0.01$ en ambos). Las razones de apariencia también muestran una importante y significativa correlación positiva con el fitness $(r$ $=.64 ; \mathrm{p}<0.01)$ y la competencia $(r=.51 ; \mathrm{p}<$ $0.01)$. Le sigue la subescala social que se relaciona de manera positiva y significativa con el fitness $(r=.54 ; \mathrm{p}<0.01)$ y la competencia $(r=$ $.59 ; \mathrm{p}<0.01)$, para finalizar con el fitness que lo hace con la competencia $(r=.75 ; \mathrm{p}<0.01)$.

Tabla 2.- Media $(M)$, desviación típica $(D T)$, coeficiente alfa $(\alpha)$, curtosis, asimetría y correlaciones de todas las subescalas.

\begin{tabular}{|c|c|c|c|c|c|c|c|c|c|c|}
\hline Subescalas & M & DT & $\alpha$ & Curtosis & Asimetría & 1 & 2 & 3 & 4 & 5 \\
\hline 1.- Disfrute & 5.83 & .86 & .84 & -.31 & -.62 & - & $.34 * *$ & $.79 * *$ & $.67 * *$ & $.79 * *$ \\
\hline 2.- Apariencia & 4.45 & 1.57 & .89 & -.84 & -.39 & - & - & $.16^{*}$ & $.64 * *$ & $.51 * *$ \\
\hline 3.- Social & 5.85 & .94 & .77 & .48 & -.85 & - & - & - & $.54 * *$ & $.59 * *$ \\
\hline 4.- Fitness/Salud & 5.57 & 1.05 & .80 & .22 & -.73 & - & - & - & - & $.75 * *$ \\
\hline 5.- Competencia & 5.68 & 1.03 & .88 & -.22 & -.64 & - & - & - & - & - \\
\hline
\end{tabular}




\section{4.- DISCUSIÓN.}

El análisis descriptivo de las diferentes subescalas muestra que las personas dan mayor importancia a los motivos relacionados con las relaciones sociales así como al placer y la diversión como motores de la participación en actividades físicas, lo que concuerda con los resultados hallados por Moreno y Gutiérrez (1998) en un estudio que realizaron con usuarios de instalaciones acuáticas, donde los principales motivos de práctica eran el disfrute, las relaciones sociales, la salud y la forma física. Del mismo modo, también son similares a los encontrados en 1984 por García Ferrando (1990), ya que la mayoría de los universitarios españoles realizaban ejercicio físico por motivos como divertirse y pasar el tiempo libre, porque les gustaba el deporte, por hacer y encontrarse con las amistades, por evasión, por mantener la línea, competir y con menor importancia, por hacer carrera profesional como deportista y a los hallados por Ponseti, Gili, Palou y Borrás (1998) donde la diversión y el ocio junto a la salud y la forma física eran las principales razones de los estudiantes de secundaria.

Los resultados encontrados por García Ferrando $(2001,2006)$ en la población española a través de la encuesta sobre hábitos deportivos reflejan que la diversión, el gusto por el deporte en sí y el hacer ejercicio físico, siguen siendo los motivos más frecuentes a la hora de practicar actividades físicas y deportivas, llegando a no variar su importancia desde 1990 hasta la última encuesta realizada en 2005.

El segundo grupo de razones en importancia por las que afirman practicar actividades físicas está compuesto por las derivadas de la competencia y el mantenimiento y mejora de la salud. Estos resultados que reflejan la importancia de la salud, coinciden con los encontrados por Fraile y De Diego (2006) en España, Italia, Francia y Portugal, donde los resultados evidenciaron que un amplio porcentaje de niños y adolescentes participaron en actividades deportivas por mejorar su salud.

Por otro lado, diferentes investigaciones como la realizada por Piéron, Telama, Almond y Carreiro da Costa (1999), Castillo, Balaguer y Duda (2000), López y Márquez (2001), Sit, Kerr y Wong (2008) y Gómez-López, Ruiz, García, Granero y Piéron (2009) han puesto de manifiesto que el placer y la salud son dos de los principales motivos para practicar actividad física. Estas razones se relacionan con la búsqueda de bienestar y calidad de vida, y favorecen la adherencia al ejercicio físico.

Es más, Frederick-Recascino y SchusterSmith (2003) indicaron que las razones de diversión, competencia y relaciones sociales estaban más relacionadas con la adherencia al ejercicio físico que la apariencia o el fitness.

Finalmente, el grupo de motivos menos valorado por la población objeto de estudio fue la apariencia, lo que viene a contradecir la importancia que ha ido adquiriendo valores como la estética en la sociedad posmoderna y los resultados de anteriores estudios como el realizado por Olmand, Daley y Richard (2004) ó Kilpatrick, Hebert y Bartholomew (2005), donde se resalta la importancia de la preocupación por la imagen corporal como motor para la participación en actividades físicas.

Por lo tanto una implicación práctica que deriva de los resultados obtenidos es que para este grupo de personas, habría que promover la práctica de actividades físicas no competitivas donde las relaciones sociales y la diversión sean los principales pilares y estas actividades estén orientadas a la mejora de la salud y la competencia. En esta línea, Martínez, Hellín, Pavón y Moreno (2010) proponen las siguientes estrategias y actividades: ofertar mayor número de prácticas grupales, que los propios usuarios en pequeños grupos elaboren sesiones completas o partes de la misma siguiendo siempre las directrices del técnico, fomentar actividades grupales fuera del centro, desarrollar estrategias para el desarrollo de la conducta autodeterminada y la motivación intrínseca e integrar a aquellas personas que más se suelen aislar en las actividades de grupo.

\section{5.- CONCLUSIONES.}

Se puede afirmar que la escala MPAM-R es un instrumento válido y fiable en la medición de los motivos de práctica en el ámbito de la actividad física no competitiva.

Los resultados reflejan que los principales motivos que impulsan la práctica de actividades físicas son las relaciones sociales y la diversión, seguidos de otros como la salud y la competencia, para finalizar con la apariencia como razón menos valorada.

En cuanto a las futuras líneas de investigación que se derivan de este estudio, sería interesante profundizar en los motivos de práctica 
en función del análisis de las variables sociodemográficas edad y sexo de los practicantes.

Con respecto a las limitaciones del estudio, podemos destacar que aunque la presente investigación amplía la información proporcionada previamente por investigaciones similares, hay que reconocer que el tamaño de la muestra analizada limita la generalización de los resultados, por lo que estos deben considerarse como preliminares, necesitando de una futura replicación.

\section{6.- REFERENCIAS BIBLIOGRÁFICAS.}

Bakker, F.C., Whiting, H.T.A., Van der Brug, H. (1990). Sport psychology: Concepts and appliations. Chichester: John Wiley \& Sons.

Balaguer, I. (1994). Entrenamiento psicológico del deporte. Valencia: Albatros.

Bollen, K. \& Long, J. S. (1994). Testing structural equation models. Newbury Park, CA: Sage.

Canton, E., Mayor, L. \& Pallarés, J. (1995). Factores motivacionales y afectivos en la iniciación deportiva. Revista de Psicología General y Aplicada, 48, 59-75.

Castillo, I., Balaguer, I. \& Duda, J.L. (2000). Las orientaciones de meta y los motivos de práctica deportiva en jóvenes deportistas valencianos escolarizados. Revista de Psicología del Deporte, 9(1-2), 37-50

Cervelló, E., Escartí, A. \& Guzmán, J.F. (2007). Youth sport dropout from the achievement goal theory. Psicothema, 19(1), 65-71.

Dosil, J. (2008). Psicología de la Actividad Física y del Deporte (2 ${ }^{a}$ edición). Madrid: McGraw-Hill.

Escartí, A. \& Brustad, R. (2002). Estudio de la motivación deportiva desde la perspectiva de la teoría de metas. En J. Dosil (ed.), Psicología y rendimiento deportivo (pp. 57-70). Ourense: Gersam.

Escartí, A. \& Cervelló, E. (1994). La motivación en el deporte. En I. Balaguer (Ed.), Entrenamiento psicológico en deporte: Principios y aplicaciones (pp. 61-90). Valencia: Albatros Educación.

Fraile, A. \& De Diego, R. (2006). Motivaciones de los escolares europeos para la práctica del deporte escolar. Un estudio realizado en España, Italia, Francia y Portugal. Revista Internacional de Sociología, 44, 85-109.

Frederick- Recascino, C.M. \& Ryan, R.M. (1993). Differences in motivation for sport and exercise and their relations with participation and mental health. Journal of Sport Behavior, 16(3), 124146.
Frederick- Recascino, C.M. \& Schuster-Smith (2003). Competition and intrinsic motivation in physical activity: A comparison of two groups. Journal of Sport Behavior, 26(3), 240-254.

García Ferrando, M. (1990). Aspectos sociales del deporte. Una reflexión sociológica. Madrid: Alianza Editorial.

García Ferrando, M. (2001). Los españoles y el deporte: prácticas y comportamientos en la última década del siglo XX. Encuesta sobre los hábitos deportivos de los españoles, 2000. Madrid: Ministerio de Educación, Cultura y Deporte. CSD.

García Ferrando, M. (2006). Posmodernidad y Deporte: entre la individualización y la masificación. Encuesta sobre hábitos deportivos de los españoles, 2005. Madrid: Consejo Superior de Deportes. Centro de Investigaciones Sociológicas.

Gómez-López, M., Ruiz, F., García, M.E., Granero, A. \& Piéron, M. (2009). Motivaciones aludidas por los universitarios que practican actividades físico-deportivas. Revista Latinoamericana de Psicología, 41(3), 519-532.

Kilpatrick, M., Hebert, E. \& Bartholomew, J. (2005). College students motivation for physical activity: Differentiating men's and women's motives for sport participation and exercise. Journal of American College Health, 54(2), 87-94.

López, C. \& Márquez, S. (2001). Motivación en jóvenes practicantes de lucha leonesa. Revista de Psicología del Deporte, 10(1), 9-22.

Martínez, A., Hellín, P., Pavón, A. \& Moreno, J.A. (2010). Motivos de práctica físico-deportiva. En J.A. Moreno \& E. Cervelló (Coords.), Motivación en la actividad fisica y el deporte. Sevilla: Wanceulen.

Masachs, M., Puente, M. \& Blasco, T. (1994). Evolución de los motivos para participar en programas de ejercicio físico. Revista de Psicología del Deporte, 5, 71-80.

Masachs, M., Puente, M. \& Blasco, T. (1994). Evolución de los motivos para participar en programas de ejercicio físico. Revista de Psicología del Deporte, 5, 71-80.

Moreno, J.A. \& Gutiérrez, M. (1998). Intereses, actitudes y motivación hacia la práctica terrestre y acuática de los españoles. En J.A. Moreno, P.L. Rodríguez \& F. Ruiz (Eds.), Actividades acuáticas: Ámbitos de aplicación (pp. 185-204). Murcia: Universidad de Murcia.

Moreno, J.A., Cervelló, E. \& Martínez, A. (2007). Validación de la escala de Medida de los Motivos para la Actividad Física-Revisada en españoles: Diferencias por motivos de participación. Anales de Psicología, 23(1), 167-176. 
Olmand, A., Daley, A. \& Richard, N. (2004). Motives for sport and exercise in younger and older half marathon runners. Journal of Sport Sciences, 22, 310-311.

Palomares, J. (2005). Nuevas tendencias en la actividad física y deportiva: un ejemplo de la creciente diversificación y complejidad de las prácticas. Retos. Nuevas tendencias en Educación Física, Deporte y Recreación, 8, 5-8.

Pedhazur, E.J. (1982). Multiple regression in behavioral research: explanation and prediction. New York: Holt, Rinehart and Winston.

Piéron, M., Telama, R., Almond, L. \& Carreiro da Costa, F. (1999). Estilo de vida de jóvenes europeos: um estudio comparativo. Revista de Educación Física, 76, 5-13.

Ponseti, F., Gili, M., Palou, P. \& Borrás, P. (1998). Intereses, motivos y actitudes hacia el deporte en adolescentes: diferencias en función del nivel de práctica. Revista de Psicología del Deporte, 7(2), 259-274.

Roberts, G.C. (1992). Motivation in sport an exercise: Conceptual constraints and conceptual convergence. In G.C. Roberts (ed.), Motivation in sport and exercise (pp. 3-30). Champaign, Il.: Human Kinetics.
Ryan, R.M., Frederick, C.M., Lepes, D., Rubio, N. \& Sheldon, K.M. (1997). Intrinsic motivation and exercise adherence. International Journal of Sport Psychology, 28, 335-354.

Ryska, T.A., Hohensee, D., Cooley, D. \& Jones, C. (2002). Participation motives in predicting sport dropout among Australian youth gymnasts. North American Journal of Psychology, 4(2), 199-210.

Santos-Rosa, F.J., García, T., Jiménez, R., Moya, M. \& Cervelló, E. (2007). Predicción de la satisfacción con el rendimiento deportivo en jugadores de tenis: efecto de las claves situacionales. Motricidad. European Journal of Human Movement, 18, 41-60.

Sit, C.H.P., Kerr, J.H. \& Wong, I.T.F. (2008). Motives for and barriers to physical activity participation in middle-aged Chinese women. Psychology of Sport and Exercise, 9(3), 266-283.

Stevens, J. (1992). Applied multivariate statistics for the social sciences. Hillsdale, NJ: Lawrence Erlbaum.

Weinberg, R.S. \& Gould, D. (2010). Fundamentos de psicología del deporte y del ejercicio físico $\left(4^{a}\right.$ edición). Madrid: Panamericana. 